\title{
Descripción de los pacientes con hemorragia intracerebral espontánea en el Hospital San José de Bogotá
}

\author{
Jeisson Ospina Osorio', Álvaro Bedoya², Javier Patiño ${ }^{3}$ \\ 1 Residente de quinto año Neurocirugía Fundación universitaria de ciencias de la salud, Hospital San José, Hospital Infantil \\ Universitario de San José de Bogotá, Colombia. \\ 2 Residente de Primer año Neurocirugía Fundación universitaria de ciencias de la salud, Hospital San José, Hospital Infantil \\ Universitario de San José de Bogotá, Colombia. \\ 3 Instructor asistente departamento Neurocirugía, Fundación universitaria de ciencias de la salud, Hospital San José, Hospital \\ Infantil Universitario de San José de Bogotá, Colombia.
}

Rev. Chil. Neurocirugía 45: 131-135, 2019

\begin{abstract}
Resumen
Introducción: La hemorragia intracerebral espontánea (HIE) se ha convertido en una enfermedad con alta prevalencia que genera una morbilidad y mortalidad considerable y más en nuestra región, con factores de riesgo ya conocidos por la literatura mundial, pero sin definir en nuestra población las características epidemiológicas de esta patología. Objetivos: Caracterizar los pacientes con hemorragia intracerebral espontánea como a la vez medir mortalidad y puntajes de uso clínico para esta patología. Materiales y Métodos: Se realizó de corte transversal en el período comprendido de noviembre de 2013 hasta diciembre de 2016, usando como criterios de selección pacientes con diagnóstico de hemorragia intracerebral espontánea o no traumática y edad mayor de 18 años. Resultados: Se encontraron 102 pacientes, con igual porcentaje en la aparición de hemorragia intracerebral espontánea entre los hombres y las mujeres, El 38\% de los pacientes presentaron como antecedente hipertensión arterial, el 7\% recibió algún tipo de intervención quirúrgica, 12 del total fallecieron a consecuencia de la HIE y de estos solo a uno de ellos se le realizo drenaje del hematoma intraparenquimatoso y se colocó una ventriculostomia. Conclusiones: Se encontraron resultados muy similares a los reportados en los diferentes estudios a nivel mundial, se debe implementar estudios prospectivos que permitan obtener más variables y resultados que permitan impactar en esta patología.
\end{abstract}

Palabras clave: Hemorragia intracraneal hipertensiva, hemorragia cerebral, hipertensión.

\begin{abstract}
Introduction: Spontaneous intracerebral hemorrhage has become a disease with high prevalence that generates a considerable morbidity and mortality and more in our region, with risk factors and known in the world literature, but if we define in our population the epidemiological characteristics of this pathology. Objectives: Characterize patients with spontaneous intracerebral hemorrhage as well as to measure mortality and clinical use scores for this pathology. Materials and Methods: A cross-sectional descriptive observational study was conducted in the period from November 2013 to December 2016, using as criteria of selection patients with diagnosis of spontaneous or non-traumatic intracerebral hemorrhage, age over 18 years. Results: 102 patients were found, of which there was no significant difference in the appearance of spontaneous intracerebral hemorrhage among men, $38 \%$ of the patients presented as a history of arterial hypertension, $7 \%$ received one type of surgical intervention, twelve of the total A consequence of the HIE died and only 1 of them underwent drainage of the intraparenchymal hematoma and a ventriculostomy was placed. Conclusions: Prospective studies should be implemented to obtain more variables and results that allow impact on this pathology.
\end{abstract}

Key words: Intracranial hemorrhage, Hypertensive, hypertension, cerebral hemorrhage. 


\section{Introducción}

La hemorragia intracerebral espontánea (HIE) o no traumática es definida por los diferentes expertos como el inicio abrupto no traumático de cefalea, alteración de estado de conciencia y/o focalización neurológica que está asociado a hemorragia intraparenquimatosa diagnosticada por estudios imagen lógicos (topográficos o de resonancia magnética) o como también por estudio patológico (autopsia clínica) y que a la vez no es atribuible a transformación hemorrágica de un infarto cerebral ${ }^{1-3}$. La HIE da cuenta de $10-15 \%$ de todos los ictus ${ }^{1,2,4}$, incluso mostrando el doble de incidencia que la hemorragia subaracnoidea espontánea ${ }^{5}$, generando por ende en una gran parte de la población afectada por esta patología perdida de funcionalidad y en caso más severos la muerte ${ }^{1}$.

Como país referente a nuestra región esta Brasil que hasta la fecha es uno de los países más afectados a nivel latinoamericano y mundial, llegando incluso a 128 muertes por cada 100.000 habitantes anualmente ${ }^{6,7}$, cifras alarmantes cuando se compara frente a países desarrollados como España y en Estados Unidos donde la mortalidad de estos asciende a 15 casos por 100.000 habitantes/año ${ }^{4}$. En Colombia no hay estudios que detallen el comportamiento de esta enfermedad ni el impacto de la prevención primaria y secundaria como del tratamiento médico y quirúrgico de esta patología, es por esto que es necesario conocer si la población comparte los mismo factores de riesgo como los encontrados en las diferentes publicaciones, como lo son, la edad superior de los 55 años, el género masculino, la hipertensión arterial (HTA) y el alcohol ${ }^{8}$.

La hipertensión arterial es la enfermedad asociada a la hemorragia intracerebral espontánea que en las diferentes series de casos se encuentra entre el $55-81 \%$, siendo esta misma un factor desencadenante del sangrado encefálico en el $91 \%$ de los pacientes al momento del ictus sin que se pueda establecer si es reflejo patofisiológico, y en un menor porcentaje se encuentra asociado las diferentes patologías estructurales como las lesiones tumorales primarias/secundarias, la enfermedad amiloidea, lesiones vasculares y/o infecto contagiosas y la de menor frecuencia y no por ello menos impor- tante la farmacológica y por abuso de sustancias de uso recreacional $\left.\right|^{9,10}$. Por otro lado, un tercio de los pacientes con HIE no tienen ningún antecedente conocido ${ }^{1,8}$.

Como lo indican las diferentes guías clínicas internacionales la información epidemiológica de esta enfermedad es limitada ${ }^{11,12}$, siendo el objetivo primario de este estudio la caracterización de los pacientes con HIE y como objetivos secundarios medir mortalidad y puntajes de uso clínico para esta patología.

\section{Metodología}

Se realizó un estudio de corte transversal el cual se llevó a cabo mediante la revisión de la base de datos del servicio neurocirugía del Hospital de San José de la ciudad de Bogotá, en la cual se encontraron los datos de los pacientes atendidos con diagnóstico de hemorragia intracerebral espontánea en el período comprendido de noviembre de 2013 hasta diciembre de 2016.

Como criterios de selección se incluyeron pacientes con diagnóstico de hemorragia intracerebral espontánea o no traumática, edad mayor de 18 años. Se excluyeron paciente con el diagnóstico de hemorragia cerebral traumática o causada por patología fármaco-toxicológica.

Se obtuvieron variables demográficas y clínicas para caracterización de población, como la escala de Rankin modificada $^{13}$ y la escala de Intracerebral hemorrhage score ${ }^{14}$, como clasificación de la discapacidad neurológica y mortalidad a 30 días al momento del ingreso respectivamente. Los datos obtenidos se registraron en una base de datos tabulada según las variables del estudio, en Microsoft Office Excel. Posteriormente se hizo el análisis estadístico en STATAC 13; las variables de tipo cualitativo fueron descritas por medio de frecuencias absolutas y relativas. Las variables cuantitativas se comprobó su distribución con la prueba de Shapiro Wilk siendo no normales y se resumieron con medianas y rangos intercuartílicos (RIQ).

\section{Resultados}

En el período de estudio el cual fue de 38 meses ingresaron 159 pacientes con diagnóstico de hemorragia in- tracerebral espontánea, 102 de ellos cumplieron criterios de selección, en quienes se encontró que la frecuencia de aparición de HIE entre hombres y mujeres fue igual, con una mediana de edad de 65 años (RIQ: 55-74) (Tabla 1).

El $38 \%$ de los pacientes presentaron como antecedente único hipertensión (HTA), y $40 \%$ no presentó antecedentes de importancia. En cuanto a la localización anatómica, el 36\% se localizó en ganglios de la base, y un tercio de los pacientes presentó hemorragia intracerebral lobar; el sangrado talámico, en cerebelo y tallo fueron las de menor aparición (Tabla 1).

La estancia hospitalaria en estos pacientes tuvo una mediana de 8 días (RIQ: 4-14) y el 7\% recibió algún tipo de intervención quirúrgica siendo la de mayor realización el drenaje del hematoma intraparenquimatoso más colocación de una ventriculostomia $(n=5)$.

Doce pacientes del total fallecieron a consecuencia de la HIE y de estos solo a uno se le practicó un procedimiento neuroquirúrgico (drenaje del hematoma intraparenquimatoso y colocación de ventriculostomia).

Dentro de las escalas clínicas observadas, la escala de Rankin modificada (Figura 1), mostro que el $58 \%$ de los pacientes con HIE presentaron discapacidad leve y moderada, mientras que el $7 \%$ de los pacientes fue moderadamente severa y severa. El puntaje de HIE (ICH score) mostro que en 42 pacientes el puntaje fue de 2 lo que representa para estos paciente una probabilidad de aumento de mortalidad de $26 \%$ a los 30 días (Figura 2).

\section{Discusión}

La hemorragia intracerebral espontánea muestra en las últimas décadas un aumento en su prevalencia e incidencia, por una parte, debido al aumento de estilos de vida no saludable y al mayor reporte en el diagnóstico de esta $^{1,15}$. Siendo mayor en los países latinoamericanos dada la pobre cobertura de los programas prevención primaria así como el bajo seguimiento en la población objeto con factores de riesgo para presentar esta patología, teniendo fallas e hipertensión que como bien se conoce y la prevención primaria y secundaria de patologías como la hipertensión arterial lo que se ve reflejado 


\begin{tabular}{|c|c|}
\hline Edad Mediana (RIQ) & $65(55-74)$ \\
\hline $\begin{array}{l}\text { Género } \mathbf{n}(\%) \\
\text { Masculino } \\
\text { Femenino }\end{array}$ & $\begin{array}{l}51(50 \%) \\
51(50 \%)\end{array}$ \\
\hline $\begin{array}{l}\text { Antecedentes más frecuentes } \mathbf{n}(\%) \\
\text { Hipertensión arterial (HTA) } \\
\text { HTA y enfermedad renal cronica } \\
\text { DM tipo } 2 \\
\text { HTA y DM tipo } 2 \\
\text { Malformación arteriovenosa } \\
\text { Otros (leucemia sobreanticoagulación, trombocitopenia y trombofilia) } \\
\text { Sin antecedente }\end{array}$ & $\begin{aligned} & 39(38 \%) \\
& 2(1 \%) \\
& 1(1 \%) \\
& 6(6 \%) \\
& 3(3 \%) \\
& 11(11 \%) \\
& 40(40 \%)\end{aligned}$ \\
\hline $\begin{array}{l}\text { Lateralidad n (\%) } \\
\text { Derecha } \\
\text { Izquierda }\end{array}$ & $\begin{array}{l}51(50 \%) \\
51(50 \%)\end{array}$ \\
\hline $\begin{array}{l}\text { Localización n (\%) } \\
\text { Ganglio basal } \\
\text { Lobar } \\
\text { Talámica } \\
\text { Cerebelo } \\
\text { Tallo* }\end{array}$ & $\begin{array}{c}37(36 \%) \\
32(31 \%) \\
20(20 \%) \\
12(11 \%) \\
2(2 \%)\end{array}$ \\
\hline $\begin{array}{l}\text { Drenaje ventricular } \mathbf{n}(\%) \\
\mathrm{Si} \\
\mathrm{No}\end{array}$ & $\begin{array}{l}19(18 \%) \\
83(82 \%)\end{array}$ \\
\hline Estancia hospitalaria (días) Mediana (RIQ) & $8(4-14)$ \\
\hline $\begin{array}{l}\text { Procedimiento } \mathbf{n}(\%) \\
\text { Drenaje más ventriculostomia } \\
\text { Ventriculostomia } \\
\text { No procedimiento }\end{array}$ & $\begin{array}{c}5(5 \%) \\
2(2 \%) \\
95(93 \%)\end{array}$ \\
\hline $\begin{array}{l}\text { Mortalidad n(\%) } \\
\text { Si } \\
\text { No }\end{array}$ & $\begin{array}{l}12(12 \%) \\
90(88 \%)\end{array}$ \\
\hline
\end{tabular}

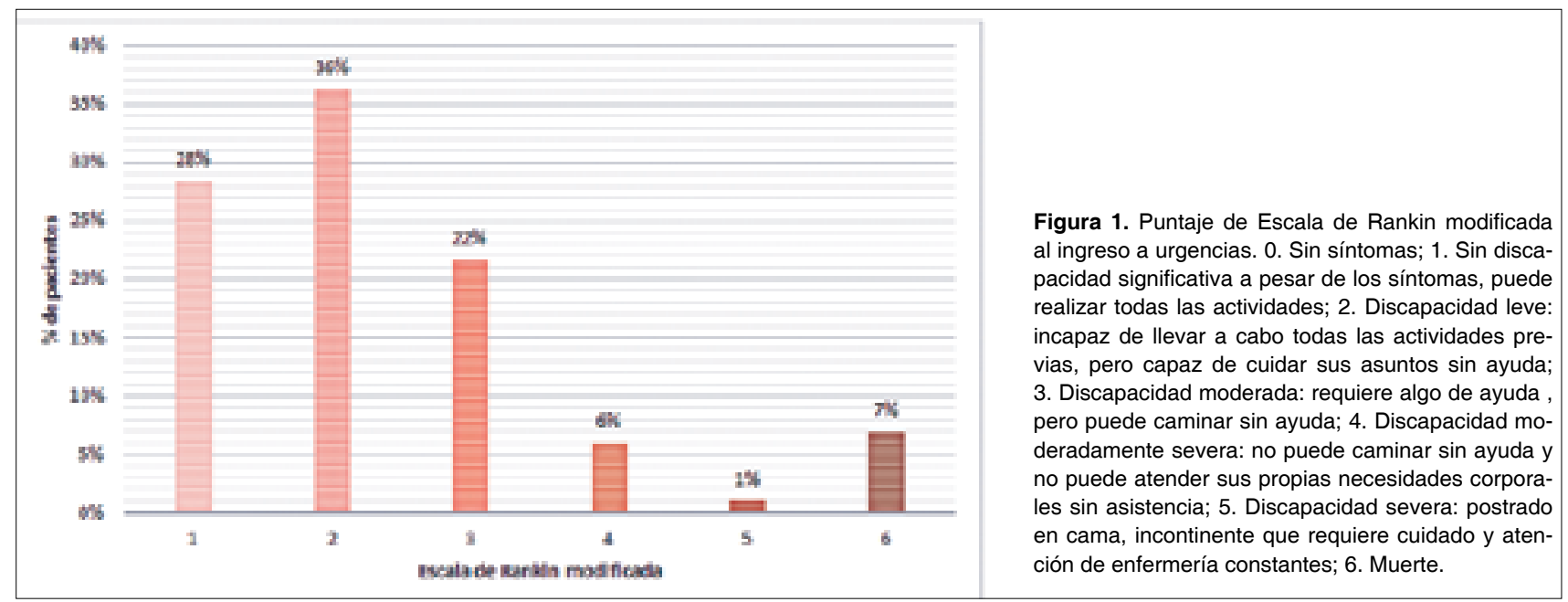




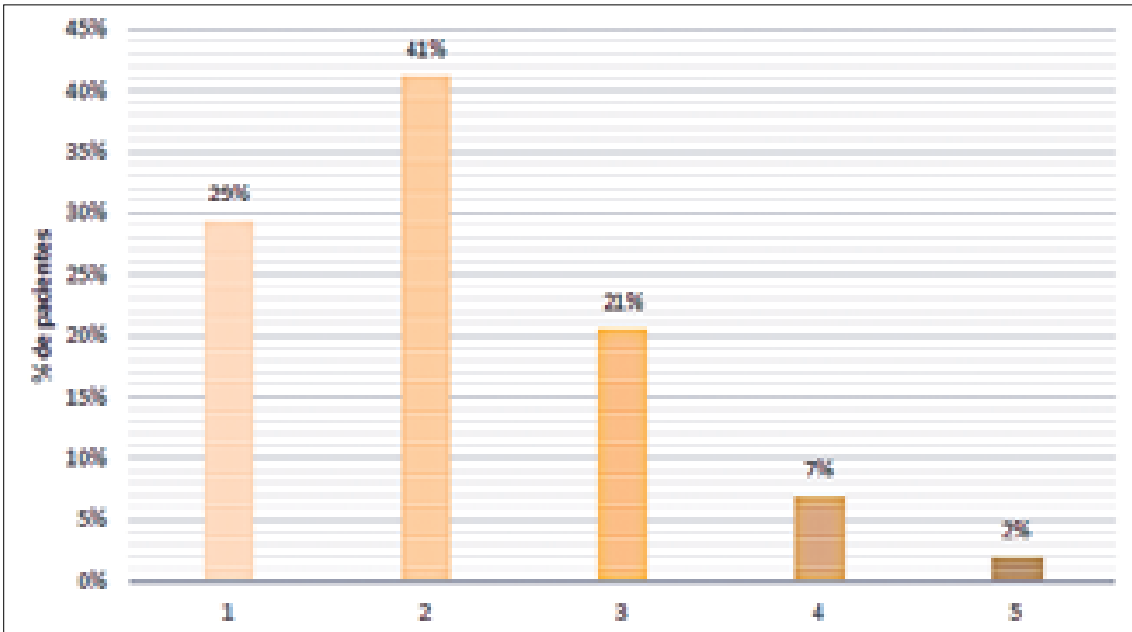

Figura 2. Puntaje de $\mathrm{ICH}$ score al ingreso a urgencias. La mortalidad a los 30 días se incrementa a razón del incremento del puntaje. ICH score 0: Sin mortalidad; ICH score; 1: $13 \%$; ICH score; 2: $26 \%$; ICH score; 3: 72\%; ICH score; 4: 97\%; ICH score, 5: $100 \%$.

en recientes publicaciones siendo el principal factor asociado y desencadenante de la $\mathrm{HIE}^{6,7}$.

Lo encontrado en este estudio no es divergente a los resultados mostrados por lo diferentes estudios latinoamericanos, norteamericanos y europeos, se encontró una mortalidad del $12 \%$ durante toda la estancia hospitala- ria muy cercana a lo referenciado por Samphron et $\mathrm{al}^{16}$, encontramos que hipertensión arterial y la localización de los sangrados, fue diferente, se encontró como antecedentes único la HTA en un $38 \%$ y sumada a otras patologías un $7 \%$ y sin referir ningún antecedente hasta el $40 \%$ de esta población. Se propone realizar estudios que determi- nen si una deficiente información a los acompañantes y a los pacientes en la prevención primaria secundaria de la HTA es determinante en el desarrollo posterior de HIE y se espera que la hipertensión arterial sea descrita como antecedente principal único o acompañado de otros.

Partiendo de la fisiopatología de la hemorragia intracerebral espontanea de origen hipertensivo se encontró una relación proporcional entre la localización ganglio basal y el antecedente de HTA, en 37 pacientes el sangrado se localizo en los ganglios de la base, ubicación predilecta de HIE causada por cifras tensionales altas, de igual manera nos interrogamos que la angiopatía amiloide cerebral se encuentre subdiagnosticada $^{9,15,17}$

Es importante resaltar que los diferentes puntajes clínicos para HIE se encuentran en rango que expresan discapacidad leve a moderada y porcentajes de mortalidad a 30 días menores al $26 \%$ en la mayoría de los pacientes. Estudios como este son el pilar para estudios prospectivos, y de mayor extensión, deben implementarse medidas de salud pública para disminuir factores de riesgo como la HTA.

Recibido: 18 de noviembre de 2018 Aceptado: 28 de diciembre de 2018

\section{Referencias}

1. Hemphill JC, 3rd, Greenberg SM, Anderson CS, Becker K, Bendok BR, Cushman M, et al. Guidelines for the Management of Spontaneous Intracerebral Hemorrhage: A Guideline for Healthcare Professionals From the American Heart Association/American Stroke Association. Stroke. 2015;46(7):2032-60.

2. Jauch EC, Pineda JA, Hemphill JC. Emergency Neurological Life Support: Intracerebral Hemorrhage. Neurocrit Care. 2015;23 Suppl 2:S83-93.

3. Qureshi Al, Tuhrim S, Broderick JP, Batjer HH, Hondo H, Hanley DF. Spontaneous intracerebral hemorrhage. N Engl J Med. 2001:344(19):1450-60.

4. Escudero AD, Marques Alvarez L, Taboada Costa F. [Up-date in spontaneous cerebral hemorrhage]. Med Intensiva. 2008;32(6):282-95.

5. Broderick JP, Brott T, Tomsick T, Miller R, Huster G. Intracerebral hemorrhage more than twice as common as subarachnoid hemorrhage. J Neurosurg. 1993;78(2):188-91.

6. Lotufo PA. Stroke in Brazil: a neglected disease. Sao Paulo Med J. 2005;123(1):3-4

7. Barros JB, Goulart AC, Alencar AP, Lotufo PA, Bensenor IM. The influence of the day of the week of hospital admission on the prognosis of stroke patients. Cad Saude Publica. 2013;29(4):769-77.

8. Ariesen MJ, Claus SP, Rinkel GJ, Algra A. Risk factors for intracerebral hemorrhage in the general population: a systematic review. Stroke. 2003;34(8):2060-5.

9. Scott M. Spontaneous intracerebral hematoma caused by cerebral neoplasms. Report of eight verified cases. J Neurosurg. 1975;42(3):33842.

10. Little JR, Dial B, Belanger G, Carpenter S. Brain hemorrhage from intracranial tumor. Stroke. 1979;10(3):283-8.

11. Zahuranec DB, Gonzales NR, Brown DL, Lisabeth LD, Longwell PJ, Eden SV, et al. Presentation of intracerebral haemorrhage in a community. J Neurol Neurosurg Psychiatry. 2006;77(3):340-4.

12. Flaherty ML, Woo D, Haverbusch M, Sekar P, Khoury J, Sauerbeck L, et al. Racial variations in location and risk of intracerebral hemorrhage. Stroke. 2005;36(5):934-7.

13. van Swieten JC, Koudstaal PJ, Visser MC, Schouten HJ, van Gijn J. Interobserver agreement for the assessment of handicap in stroke patients. Stroke. 1988;19(5):604-7. 
14. Hemphill JC, 3rd, Bonovich DC, Besmertis L, Manley GT, Johnston SC. The ICH score: a simple, reliable grading scale for intracerebral hemorrhage. Stroke. 2001;32(4):891-7.

15. Naidech AM. Diagnosis and Management of Spontaneous Intracerebral Hemorrhage. Continuum (Minneap Minn). 2015;21(5 Neurocritical Care):1288-98.

16. Sampron N, Mendia A, Azkarate B, Alberdi F, Arrazola M, Urculo E. Early mortality in spontaneous supratentorial intracerebral haemorrhage. Neurocirugia (Astur). 2010;21(2):93-8.

17. Greenberg SM, Charidimou A. Diagnosis of Cerebral Amyloid Angiopathy: Evolution of the Boston Criteria. Stroke. 2018;49(2):491-7.

Correspondencia a:

Jeisson Ospina Osorio, MD.

Calle 10 No. 18-75.

Servicio de Neurocirugía, consultorio 210. Bogotá D.C., Colombia.

Teléfono: (+057) 3538000 ext 144

jospina@fucsalud.edu.co 